\title{
Problems of Numerical Modeling on the Basis of Some Modifications of the Godunov's Scheme
}

\author{
Yu.V. Tunik \\ Institute of Mechanics, Lomonosov Moscow State University, \\ Moscow, 119192, Russia \\ tunik@imec.msu.ru
}

\begin{abstract}
The paper verifies the ability of previously proposed modifications of the Godunov's scheme develop physically justified numerical solutions of the inviscid gas dynamics equations. The modifications constructed using the approach proposed by Kolgan to improve the accuracy of solutions on spatial variables are being considered. As test it is being solved the problems of flow around of semi-infinite rectangle and of a circular cylinder by a coaxial supersonic flow. The calculations are performed on a rectangular uniform grid. It is shown that the numerical solution can correspond both to stationary and a pulsating flow around with different shape of the shock waves in front of the end wall (see figure below) depending on the viscosity of the numerical scheme and the initial parameter distribution.
\end{abstract}

Keywords: numerical scheme, Euler equations, perfect gas, supersonic flow around, entropy, total enthalpy, bow shock wave, pulsating regime, local inaccuracy.
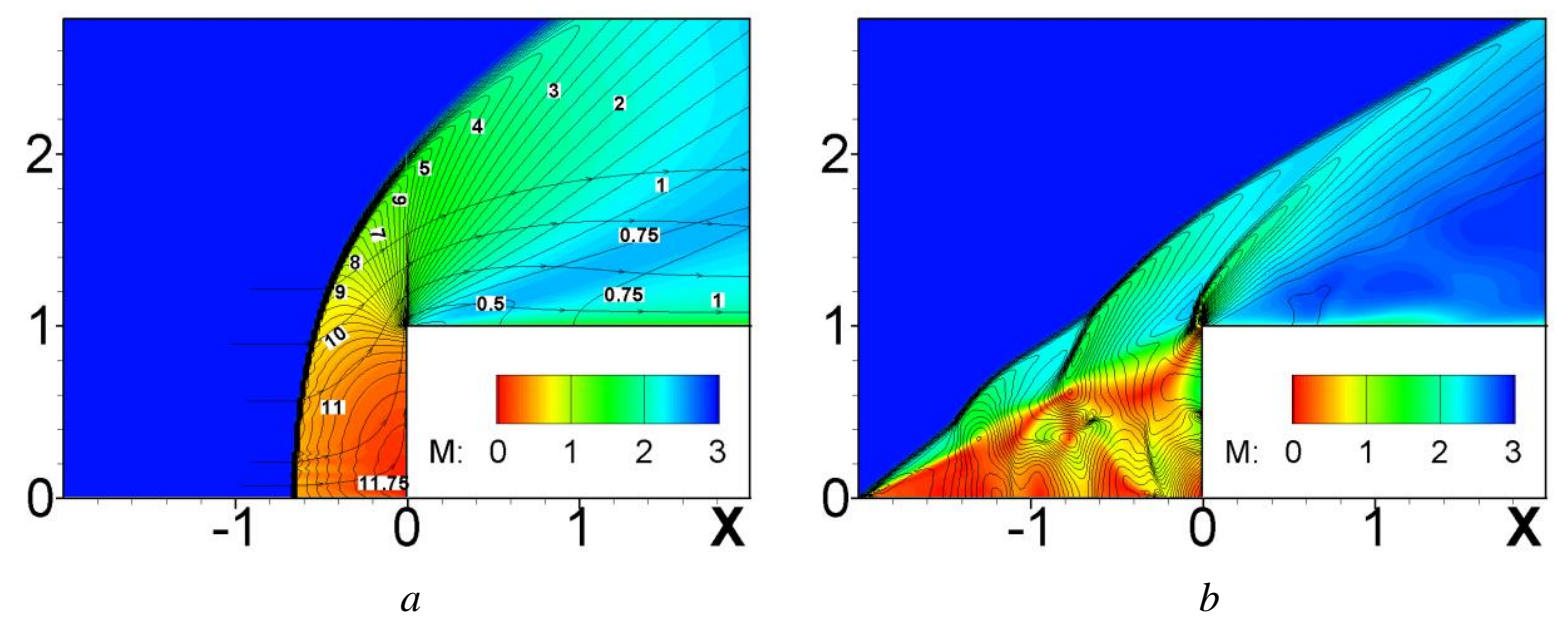

Isobars on the background of Mach number at flow around circle cylinder by supersonic coaxial flow with Mach number of 3: $a$ - the stationary solution by scheme of Godunov; $b$ - this is an intermediate picture of pulsating solution by Kolgan's scheme. 
УДК 533.6.01+519.6

\title{
Проблемы численного моделирования на основе некоторых модификаций схемы Годунова
}

\author{
Ю.В. Туник \\ НИИ механики, Московский Государственный Университет им. М.В. Ломоносова, \\ Россия, Москва, 119192, Мичуринский проспект д.1 \\ tunik@imec.msu.ru
}

\begin{abstract}
Аннотация
В работе проверяется способность ранее предложенных модификаций схемы Годунова вырабатывать физически оправданные численные решения уравнений динамики невязкого газа. Рассматриваются модификации, построенные с использованием подхода, предложенного Колганом для повышения точности решений по пространственным переменным. Решаются задачи обтекания плоского выступа и кругового цилиндра коаксиальным сверхзвуковым потоком. Расчеты проводятся на равномерной прямоугольной сетке. Показано, что численное решение может соответствовать как стационарному, так и пульсирующему обтеканию с разной формой ударных волн перед торцевой стенкой в зависимости от вязкости численной схемы и начального распределения параметров.
\end{abstract}

Ключевые слова: численная схема, уравнения Эйлера, совершенный газ, сверхзвуковое обтекание, энтропия, полная энтальпия, отошедшая ударная волна, пульсирующий режим, локальная погрешность.

\section{1. Введение}

Схема С.К. Годунова [1-2] ознаменовала новый этап численного моделирования в гидродинамике. Основные достоинства схемы: унификация и наглядность численного алгоритма, монотонность и неубывание энтропии, возможность моделирования разрывных решений. Позднее были предложены различные модификации схемы, направленные на повышение точности расчетов течений невязкого газа и жидкости. Как правило, эти улучшения связаны с изменением процедуры вычисления потоков на границах между соседними расчетными ячейками [4-11]. Первая модификация была предложена В.П. Колганом [3-4]. В отличие от схемы Годунова в схеме Колгана численное решение представляется не кусочно-постоянной, а кусочно-линейной функцией. Значение любого параметра численного решения приписывается только центру ячейки. На границе с соседней ячейкой его величина определяется производной этого параметра в рассматриваемой расчетной ячейке. Полученные таким образом значения используются при решении задачи о распаде разрыва на границе между соседними ячейками. Поскольку численное значение производной в ячейке определяется неоднозначно, в [3] предлагается выбирать ее наименьшее значение. На базе этого подхода в [12] построены модификации схемы Годунова, в которых предлагается определять потоки через границы соседних ячеек, решая задачу Римана для параметров, соответствующих промежуточной точке между центром и границей ячейки. Положение промежуточной точки может быть однозначно задано для всех ячеек параметром $\alpha: 0 \leq \alpha \leq 0.5$. Более общая схема предполагает экспоненциальную зависимость $\alpha$ от градиента газодинамических параметров в окрестности 
рассматриваемой расчетной ячейки: $\alpha=0.5 \exp \left(-\beta \frac{\phi_{l}^{\prime} h}{\phi}\right)$. Здесь $\phi_{l}^{\prime}$ - минимальная производная газодинамического параметра $\phi$ по направлению $\vec{l}$ от центра ячейки до середины границы, отделяющей ее от соседней ячейки; $h=|\vec{l}|$. Из выражения для переменного значения $\alpha$ следует, что при относительно малой величине градиента $\phi_{l}^{\prime}$ схема с промежуточной точкой приближается к схеме Колгана. При этом схемная вязкость и диффузия определяются малыми второго порядка относительно размера расчетной ячейки [12], то есть пренебрежимо малы. При больших градиентах, возникающих, например, в окрестности разрывов, данная модификация приближается к схеме Годунова первого порядка с коэффициентами вязкости и диффузии порядка размера расчетной ячейки. В промежуточном случае коэффициент схемной вязкости меняется в зависимости от $\alpha$. Величина $\beta$ задает скорость реакции схемы на изменение газодинамических параметров. При этом, строго говоря, схемы остаются схемами первого порядка аппроксимации по пространству, но позволяют уменьшить диффузию схемы Годунова и повысить точность решения задачи о распаде произвольного разрыва [12]. В [12] на примере уравнений, описывающих распространение плоских звуковых волн в идеальной жидкости, показано также, что модификация Колгана не гарантирует неубывания энтропии при моделировании нестационарных течений невязкого газа, то есть может приводить к физически неоправданным численным решениям. Для обеспечения монотонности схема требует малого шага по времени. Предложенные в [12] схемы Годунова - Колгана с промежуточной точкой позволяют получить физически оправданные численные решения задачи о распаде цилиндрической области высокого давления в среде с коаксиальным контактным разрывом.

В данной работе на базе уравнений Эйлера в качестве тестовых для схем ГодуноваКолгана решаются задачи обтекания плоского выступа и кругового цилиндра коаксиальным сверхзвуковым потоком.

\section{2. Решение тестовых задач}

\section{1. Постановка задачи}

Как в [4] и [13] задачи решаются в прямоугольной декартовой системе координат (X, Y) методом установления. Прямой угол, задающий контур кругового цилиндра и верхнюю половину плоского выступа, расположен в первом квадранте плоскости XY (рис. 1). Торцевая стенка располагается на оси ординат, горизонтальная линия контура параллельна оси абсцисс. За единицу длины принимается радиус $r_{0}$ цилиндра или высота верхней половины выступа. Расчетная область разбита вертикальной прямой $x=0$ на две зоны и ограничена осью абсцисс, стенками обтекаемого тела и отрезками прямых $x=x_{1}=-3, x=x_{2}=2$ и $y=5$. Расчетная сетка состоит из равных квадратных ячеек, стороны которых параллельны координатным осям и равны $1 / N_{\mathrm{T}}$, где $N_{\mathrm{T}}$ - число ячеек по торцевой стенке.

В начальный момент времени во всем поле течения задается число Маха $\mathrm{M}_{0}$ набегающего потока, а также давление $p_{0}$ и плотность $\rho_{0}$, которые принимаются за единицу, скорость потока направлена вдоль оси абсцисс во всей расчетной области. На оси абсцисс и стенках обтекаемого тела - условия непротекания. На верхнюю и правую границу, через которые поток выходит за пределы расчетной области, сносятся значения параметров из ближайших ячеек.

В основе математической модели - двумерные газодинамических уравнений Эйлера для плоского и осесимметричного течения, соответственно. Газ считается совершенным с показателем адиабаты 1.4 , время $t$ на рисунках отнесено к $t_{0}=r_{0} / U_{0}$, где $U_{0}=\sqrt{p_{0} / \rho_{0}}$, число Маха набегающего потока $\mathrm{M}_{0}=3$. 


\section{2. Обтекание плоского выступа}

В [13] не указаны размеры расчетной области, поэтому трудно определить число расчетных ячеек $N_{\mathrm{T}}$, приходящихся на торцевую стенку обтекаемого тела. Судя по рисункам $N_{\mathrm{T}}=8$. В результате расчета в [13] при $\mathrm{M}_{0}=3$ получена стационарная картина обтекания. В аналогичных условиях стационарная картина обтекания формируется и в расчетах по схеме Колгана [4-5].

Данная работа в целом подтверждает эти результаты: стационарное обтекание получено в расчетах по схеме Годунова (рис. 1,a), Колгана (рис. 1, б) и Годунова-Колгана (рис. 1, в-2). Как и в [13] отход параболической ударной волны от выступа по оси абсцисс равен, примерно, 1.5. Расположение изобар аналогично тому, что получено методом Русанова [13].

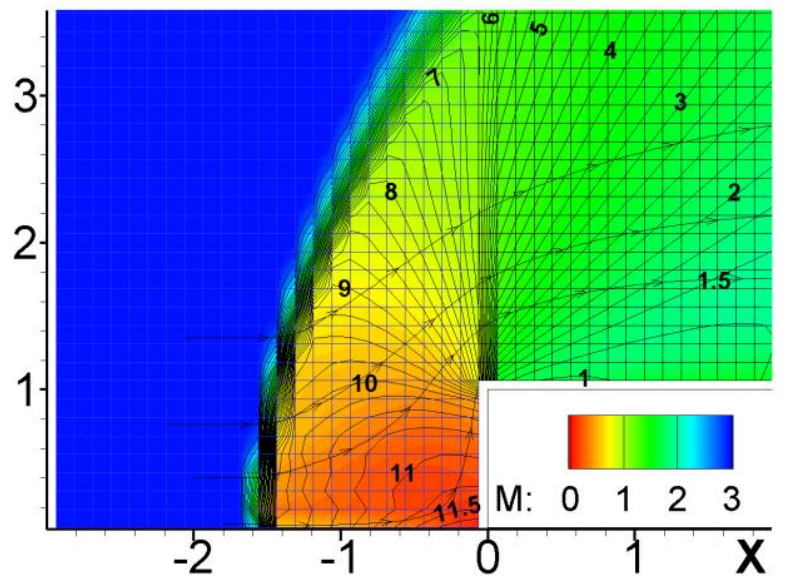

$a$

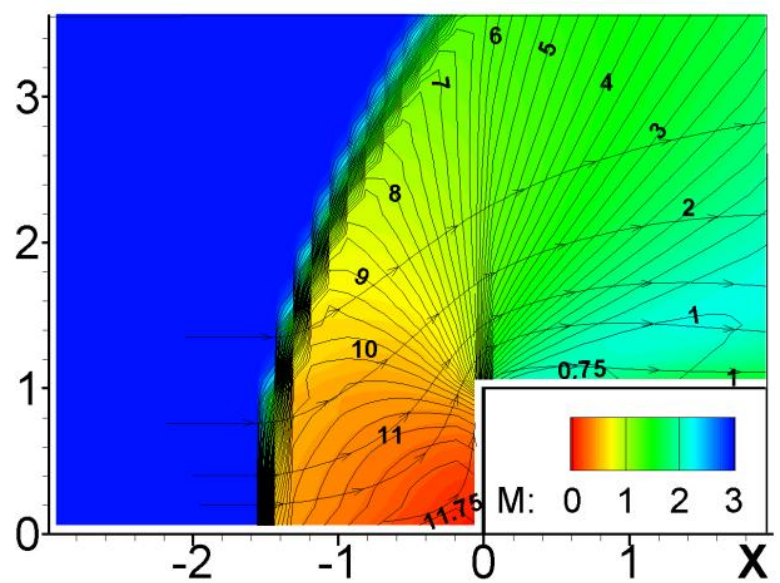

B

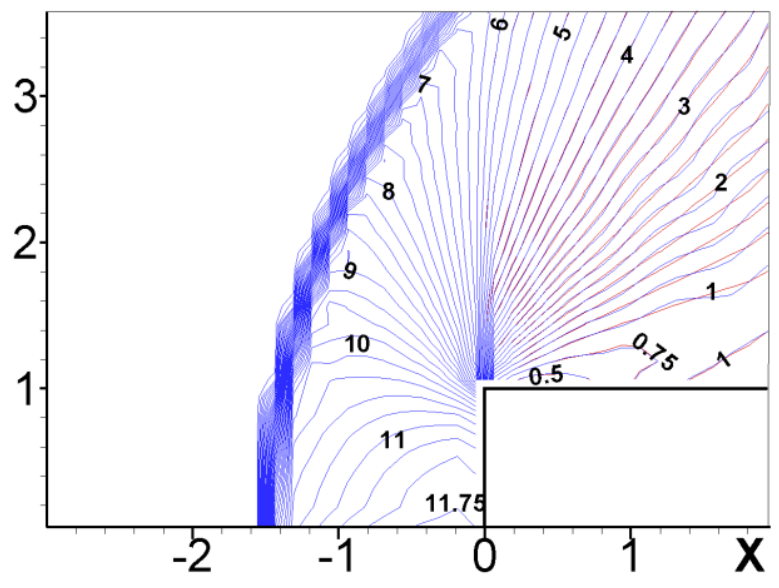

$\sigma$

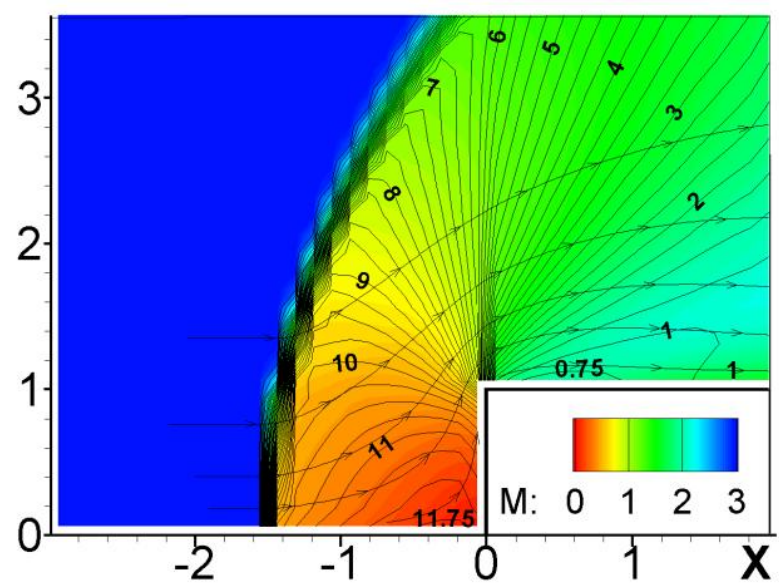

2

Рис. 1. Линии тока и изобары на фоне числа Маха в потоке: $a$ - схема Годунова при CFL $=0.4$; $\sigma$ - схема Колгана при CFL $=0.1$ и 0.4 (красные и синие линии, соответственно); 8 - схема Годунова - Колгана при $\alpha=0.4$ и $\mathrm{CFL}=0.4 ; 2-$ схема Годунова - Колгана с переменным значением $\alpha$ при $\beta=1$ и $\mathrm{CFL}=0.4$

Следует, однако, отметить извилистый характер изобар над горизонтальной стенкой выступа в расчете по схеме Колгана с числом Куранта $\mathrm{CFL}=0.4$ (рис. 1, б, синие линии). В [4] извилистый характер изобар мог остаться незамеченным из-за малой кривизны и низкой разрешающей способности плоттера. В работе [5] на приведенных рисунках область над выступом практически отсутствует. При $\mathrm{CFL}=0.1$ искривление изобар исчезает (рис. 1 , б, красные линии). С учетом этого можно предположить, что извилистый характер изобар есть следствие неабсолютной монотонности схемы Колгана, которая может быть нарушена при 
большом числе Куранта [12]. Выход на стационарное обтекание подтверждается и графиком изменения силы аэродинамического сопротивления $F$ в процессе установления (рис. $2, a$, кривая A1). Схема Годунова-Колгана с фиксированной и переменной промежуточной точкой ( $\alpha=0.4$ и $\beta=1$ на рис. 1, в и 1,2 , соответственно) дают близкие решения, более детальные, чем схема Годунова, но менее подробные, чем схема Колгана. По силе сопротивления расчеты отличаются не более, чем на $3 \%$ (рис. $2, a$ ).
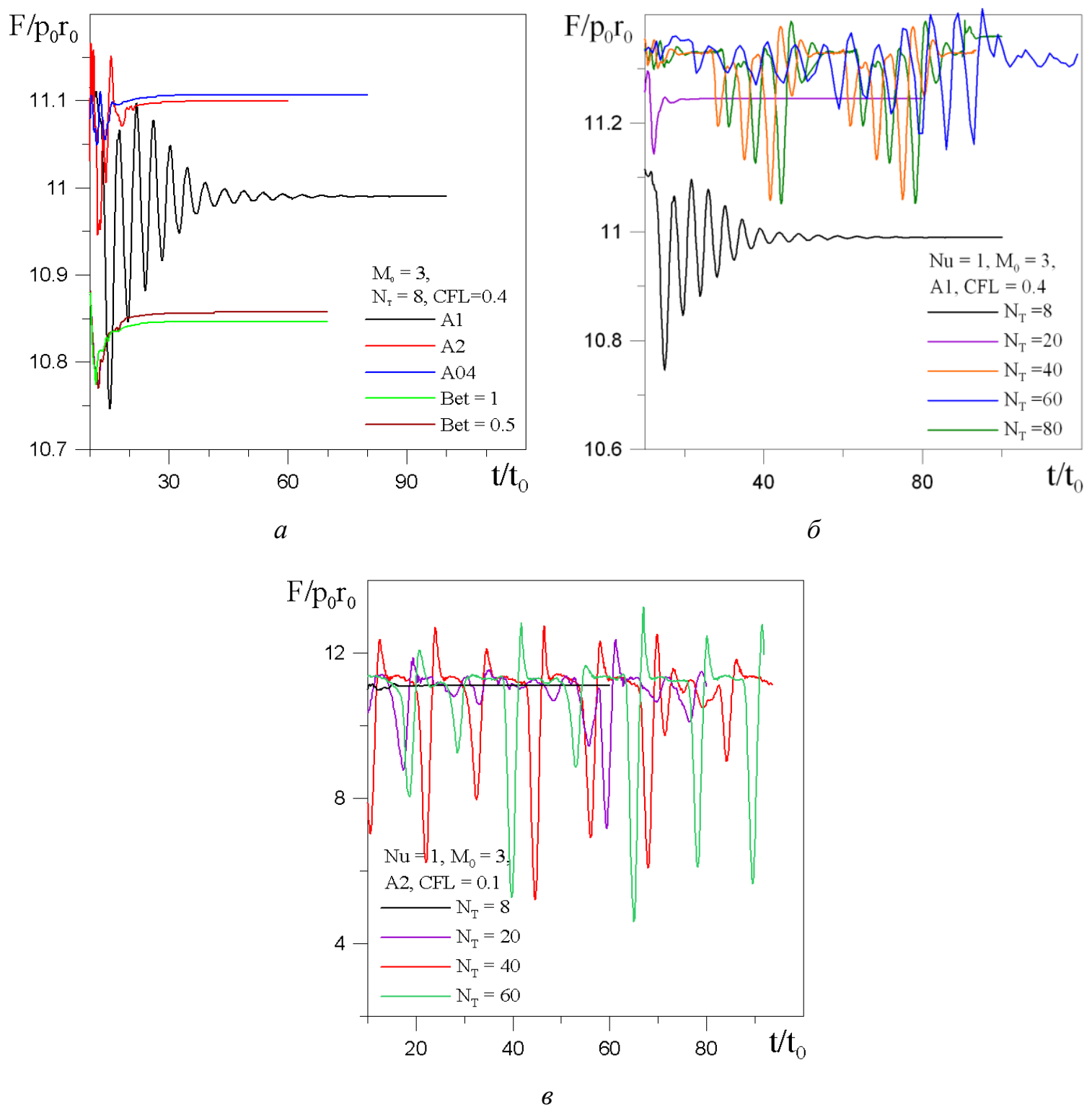

Рис. 2. Изменение силы аэродинамического сопротивления в процессе установления: $a$ - схемы Годунова (A1), Колгана (А2) и Годунова-Колгана при $\alpha=0.4$ (А04), $\beta=1$ и $\beta=0.5$ на сетке с $N_{\mathrm{T}}=8$; $\sigma$ - схема Годунова $(\mathrm{A} 1, \mathrm{CFL}=0.4) ; в$ - схема Колгана $(\mathrm{A} 2, \mathrm{CFL}=0.1)$ при разных значениях $N_{\mathrm{T}}$

На более мелкой сетке при $N_{\mathrm{T}} \geq 40$ в расчетах по схеме Годунова возникают практически незатухающие пульсации, которые на картине течения без изолиний заметить практически невозможно (рис. 3, $a$, avi_1). Не меняется и положение отошедшего скачка. Амплитуда пульсаций силы $F$ не превышает $1.5 \%$ от среднего значения (рис. 2,6 ). Пульсации являются следствием возникновения мощных возмущений перед торцевой стенкой на начальном этапе формирования решения. Со временем эти возмущения могут ослабевать и переходить в звуковые. На грубой сетке вязкость схемы быстро подавляет эти возмущения. Численное решение на мелкой сетке точнее приближает решение уравнений динамики невязкого газа, в ко- 
тором диссипативный механизм подавления пульсаций отсутствует. Подобные пульсации вряд ли можно наблюдать в эксперименте, поскольку, в частности, реальный газ обладает вязкостью.

Схема Колгана обладает меньшей вязкостью, чем схема Годунова, поэтому пульсации происходят с заметным изменением силы аэродинамического сопротивления (рис. 2, в). При этом периодически меняется структура течения, форма и положение ударной волны перед выступом (рис. 3, б, avi_2).

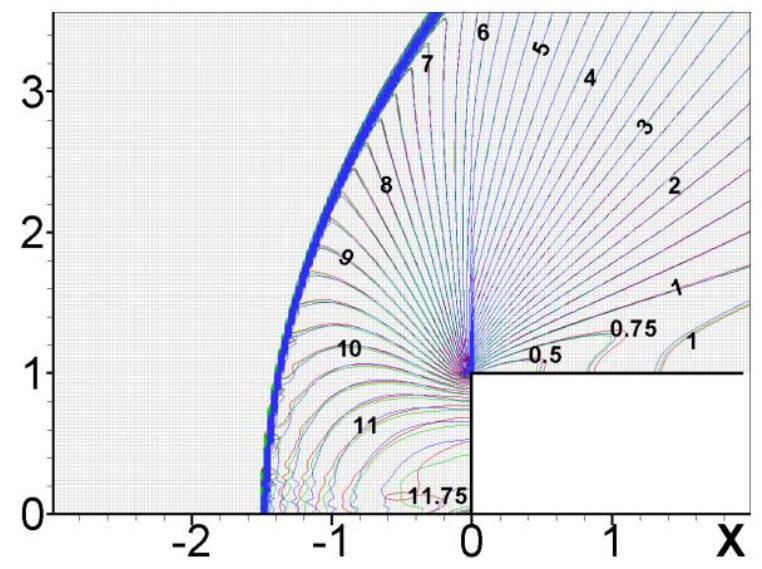

$a$

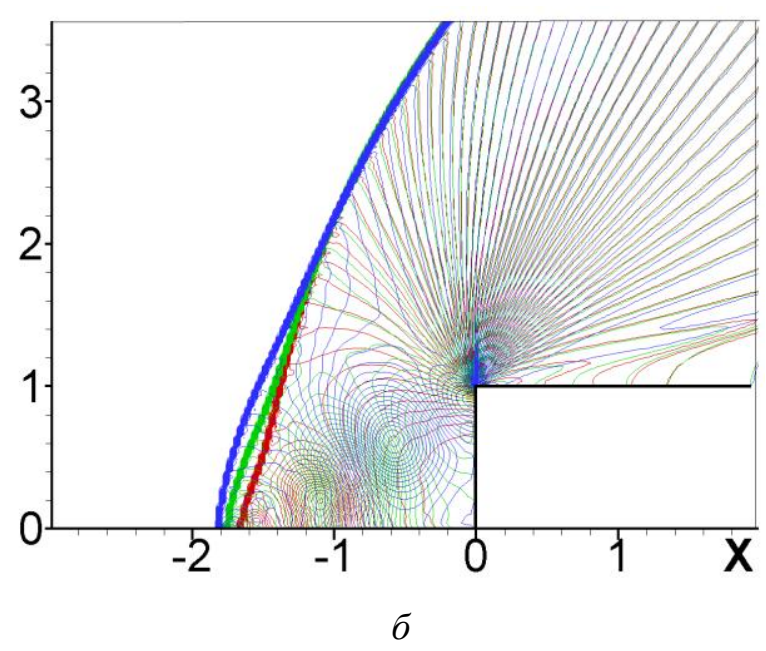

Рис. 3. Изобары, полученные в расчетах по схеме Годунова (a) и Колгана (б) при $N_{\mathrm{T}}=40$ в последовательные моменты времени: красные, зеленые и синие линии

Стационарное решение вырабатывается в расчетах по схеме Годунова-Колгана как при фиксированном, так и переменном значении $\alpha$ (рис. 4). На рисунке 5, $a$ представлено изменение аэродинамического сопротивления в случае стационарного обтекания выступа. Кривая Веt соответствует схеме с переменным значением $\alpha$ при $\beta=2$, а кривые А025 и А04 получены при $\alpha=0.25$ и 0.4. Выход на стационарное решение обеспечивает наличие эффективно работающей схемной вязкости даже при малом размере расчетных ячеек.

Несмотря на низкий уровень вязкости, схема Колгана также может привести к стационарному решению при более удачном выборе начального распределения газодинамических параметров, которое не провоцирует мощные возмущения на начальном этапе расчета (рис. 5, б, линия А2С).

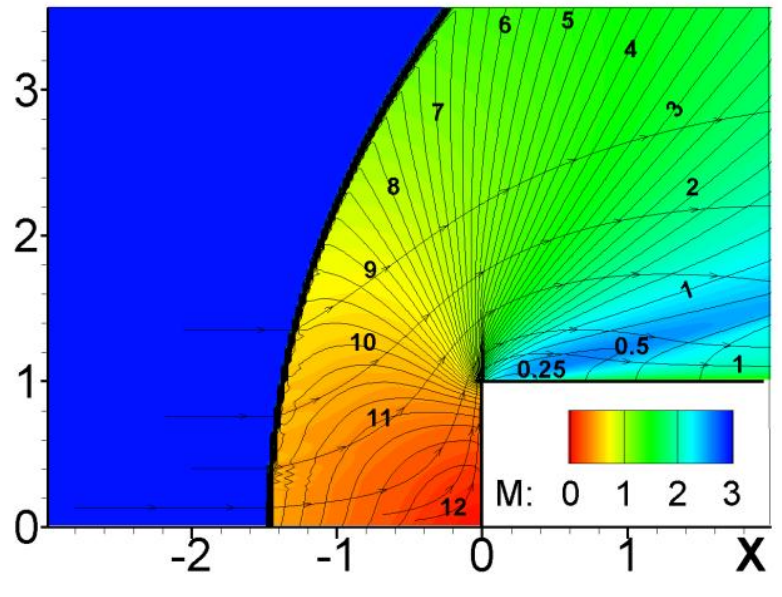

$a$

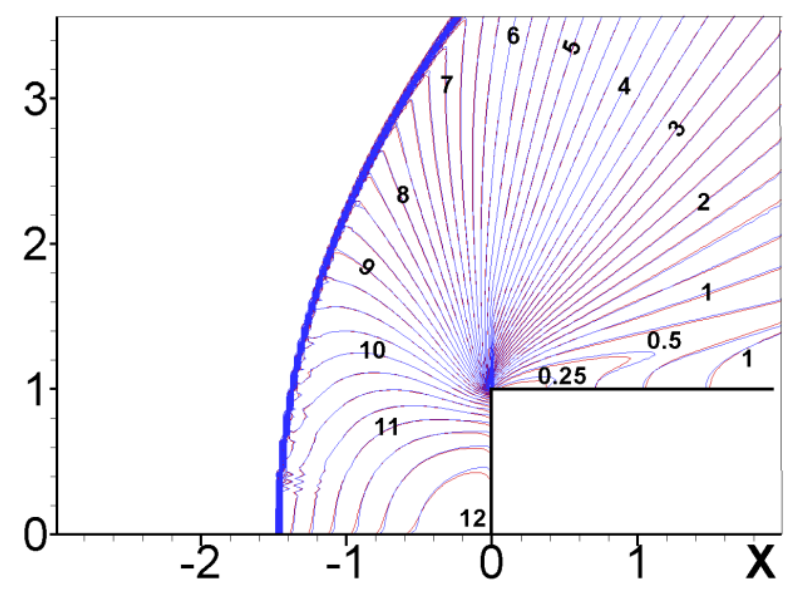

$\sigma$

Рис. 4. Стационарное обтекание выступа в расчетах по схеме Годунова-Колгана $\left(N_{\mathrm{T}}=40\right)$ : $a$ - линии тока и изобары на фоне числа Маха при $\beta=2 ; \sigma-$ изобары при $\beta=2$ и $\alpha=0.4$ (красные и синие линии, соответственно) 

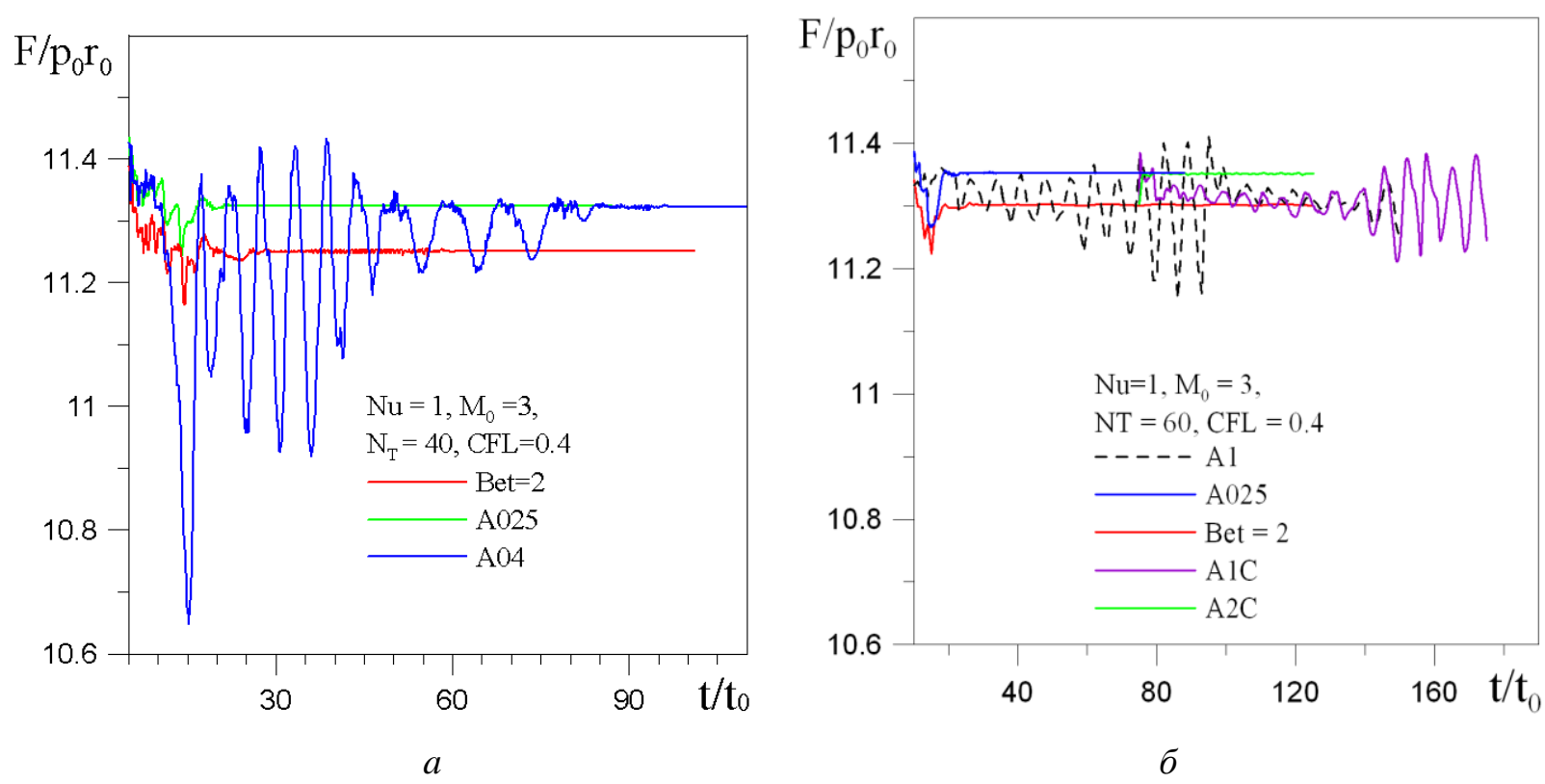

Рис. 5. Изменение во времени силы аэродинамического сопротивления при обтекании выступа: $a$ - по схемам Годунова-Колгана при $N_{\mathrm{T}}=40 ; \sigma$ - продолжение расчета по схеме Годунова (кривая А1C) и Колгана (кривая А2С) при $N_{\mathrm{T}}=60$

В этом расчете в качестве начального распределения используется стационарное решение, полученное по схеме Годунова-Колгана при $\beta=2$ и $N_{\mathrm{T}}=60$ (рис. 5, б, линия Bet=2). Аналогичное продолжение по схеме Годунова, начатое с кривой $\mathrm{Bet}=2$, восстанавливает пульсационный режим обтекания как при $\mathrm{CFL}=0.4$, так и при $\mathrm{CFL}=0.1$ (рис. 5, б, кривая A1C). Видимо, снижение точности не позволяет сохранять начальное распределение параметров, а схемной вязкости на мелкой сетке недостаточно для подавления возникающих пульсаций.

\section{3. Расчет обтекания кругового цилиндра коаксиальным сверхзвуковым потоком}

В соответствующих экспериментах обтекание цилиндра происходит в стационарном режиме с параболической отошедшей волной [14]. Соответствующие стационарные решения получены в расчетах по схеме Годунова (рис. $6, a)$ и на мелких сетках $\left(N_{\mathrm{T}} \geq 40\right)$ по схемам Годунова-Колгана при $\alpha=0.25$ и 0.4, а также при $\beta=2$ (рис. 6, $\sigma-$-, avi_3). При $\alpha=0.4$, т.e. при невысокой схемной вязкости и повышенной точности, на удалении от выступа изобары немонотонны. Оптимальной представляется схема с переменной промежуточной точкой $(\beta=2)$ : детализация картины близка к расчету при $\alpha=0.4$, но изобары монотонны. Полученная при этом картина течения качественно и количественно близка к той, что представлена в [13]: практически совпадают отход головной волны и положение внутреннего скачка SF над образующей цилиндра (рис. 6, в). Как и в [13], примерно, в 4.2 увеличивается плотность на оси симметрии при подходе к торцу и уменьшается на четверть от оси по радиусу.

При небольшом числе расчетных ячеек $\left(N_{\mathrm{T}} \leq 20\right)$ расчет по схеме Годунова - Колгана приводит к формированию решений с пульсациями, амплитуда которых на графике изменения силы аэродинамического сопротивления не превышает $2 \%$ (рис. $7, a$ ). Пульсации успешно подавляются с увеличением параметра $\beta$. Так на сетке с $N_{\mathrm{T}}=8$ при $\beta=3$ схема Годунова-Колгана дает стационарное решение (рис. 7, $a$ ).

Схема Колгана не дает однозначного ответа на вопрос о режиме обтекания цилиндра. При $N_{\mathrm{T}}=20,40$ и 80 обтекание происходит в стационарном режиме (рис. $7, \sigma$ ), а при $N_{\mathrm{T}}=8$ и 60 - с образованием пульсирующей конусообразной волны перед торцевой стенкой (рис.6, 2, avi_4). 

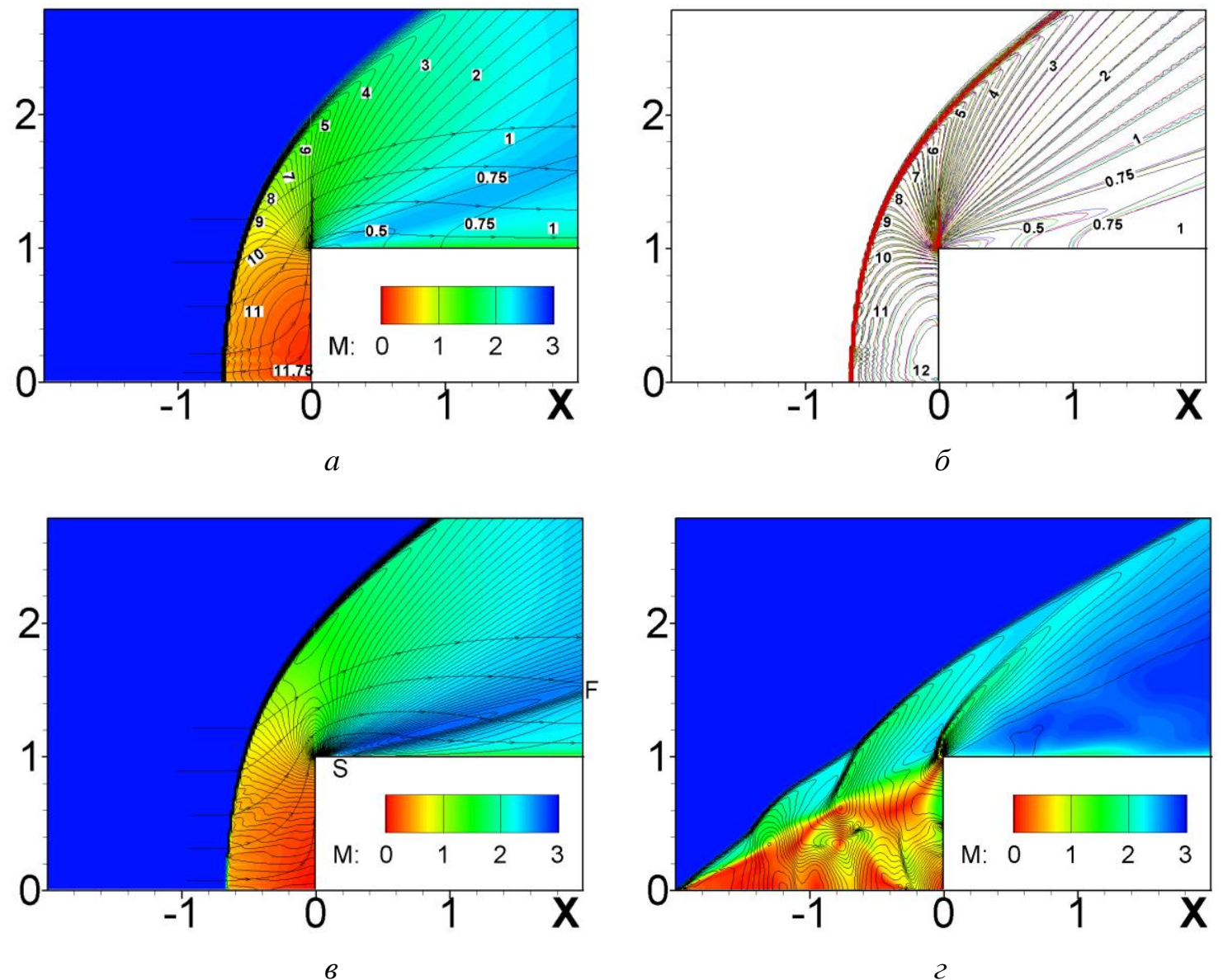

Рис. 6. Решения при $N_{\mathrm{T}}=60$ и $\mathrm{CFL}=0.1: a$ - схема Годунова (линии тока и изобары на фоне числа Маха); $\sigma$ - изобары решений по схеме Годунова-Колгана при $\alpha=0,0.25,0.4$ и $\beta=2$ (черные, зеленые, синие и красные линии, соответственно); в - линии тока и постоянного значения поперечной скорости при $\beta=2 ; 2$ - схема Колгана (мгновенное состояние пульсирующего течения)
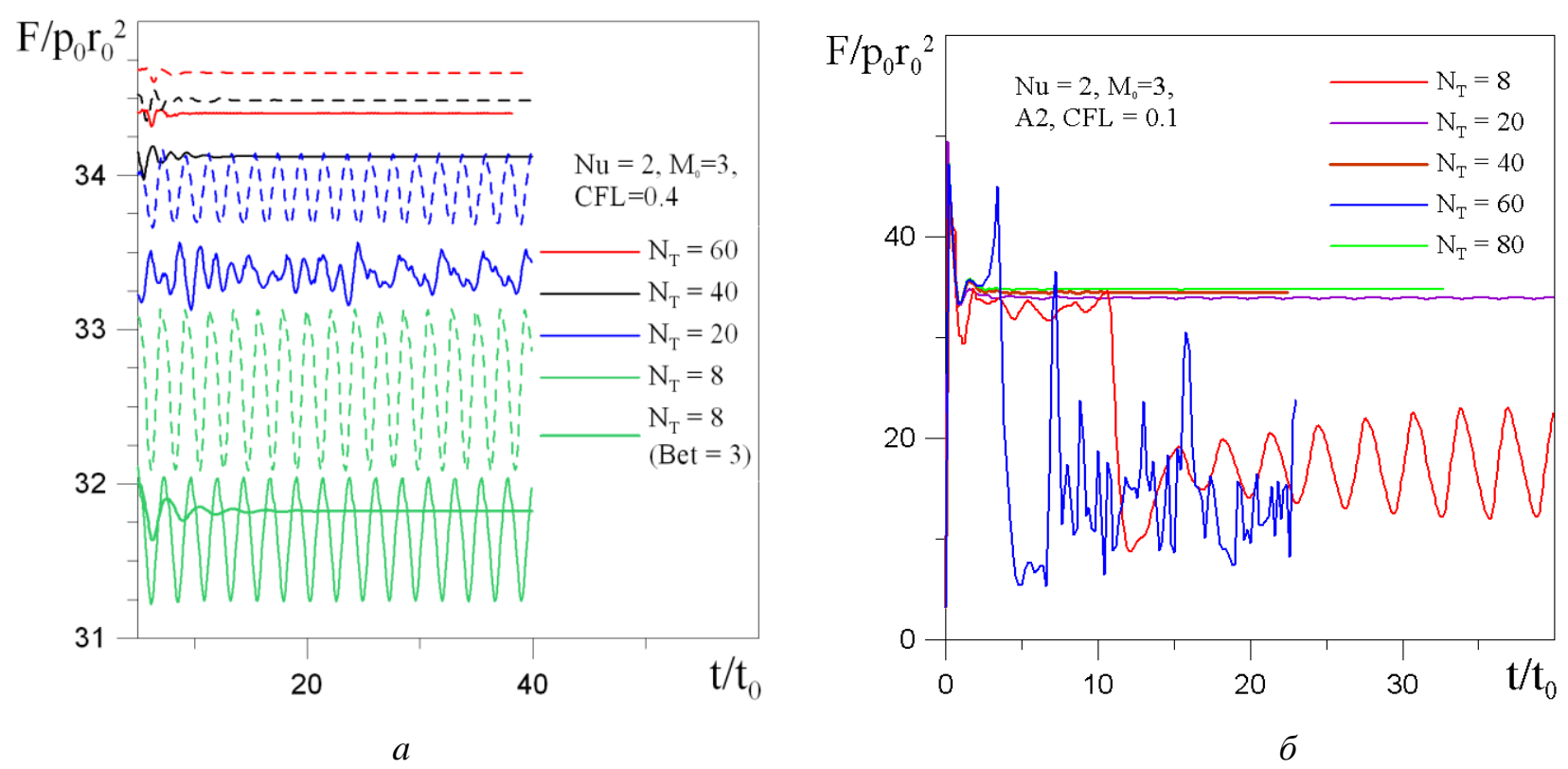

Рис. 7. Изменение во времени силы аэродинамического сопротивления цилиндра при разных значениях $N_{\mathrm{T}}: a$-схема Годунова-Колгана при $\alpha=0.25$ (штриховые линии) и $\beta=2$ (сплошные линии); $\sigma$ - схема Колгана 
Стационарные режимы обтекания цилиндра являются результатом совместного влияния диссипации энергии пульсаций, обусловленной схемной вязкостью, и конвективного выноса возмущений из области перед торцевой стенкой. Неоднозначность решения, скорее всего, связана с тем, что схема Колгана, как показано в [12], не гарантирует неубывания энтропии, а, следовательно, и однозначности решения.

\section{4. О точности расчетов}

Консервативные схемы обеспечивают достаточно малую погрешность по интегральным характеристикам: потоку массы, импульса и энергии. В случае течений в неограниченном пространстве для оценки качества численной модели естественно использовать данные о локальной погрешности, в частности, в непосредственной близости от обтекаемых объектов. На рисунке 8 представлены распределения давления и продольной скорости газа на горизонтальной поверхности рассматриваемых тел при стационарном обтекании. В случае схемы Колгана стационарные режимы получены при продолжении решений, установившихся в расчетах по схеме с промежуточной точкой. Экстремальные изменения параметров в окрестности угловой точки $(x=0)$ характерны для расчетов по схеме Колгана. Наиболее близкие результаты, как и следовало ожидать, дает схема Годунова-Колгана при $\alpha=0.4$.
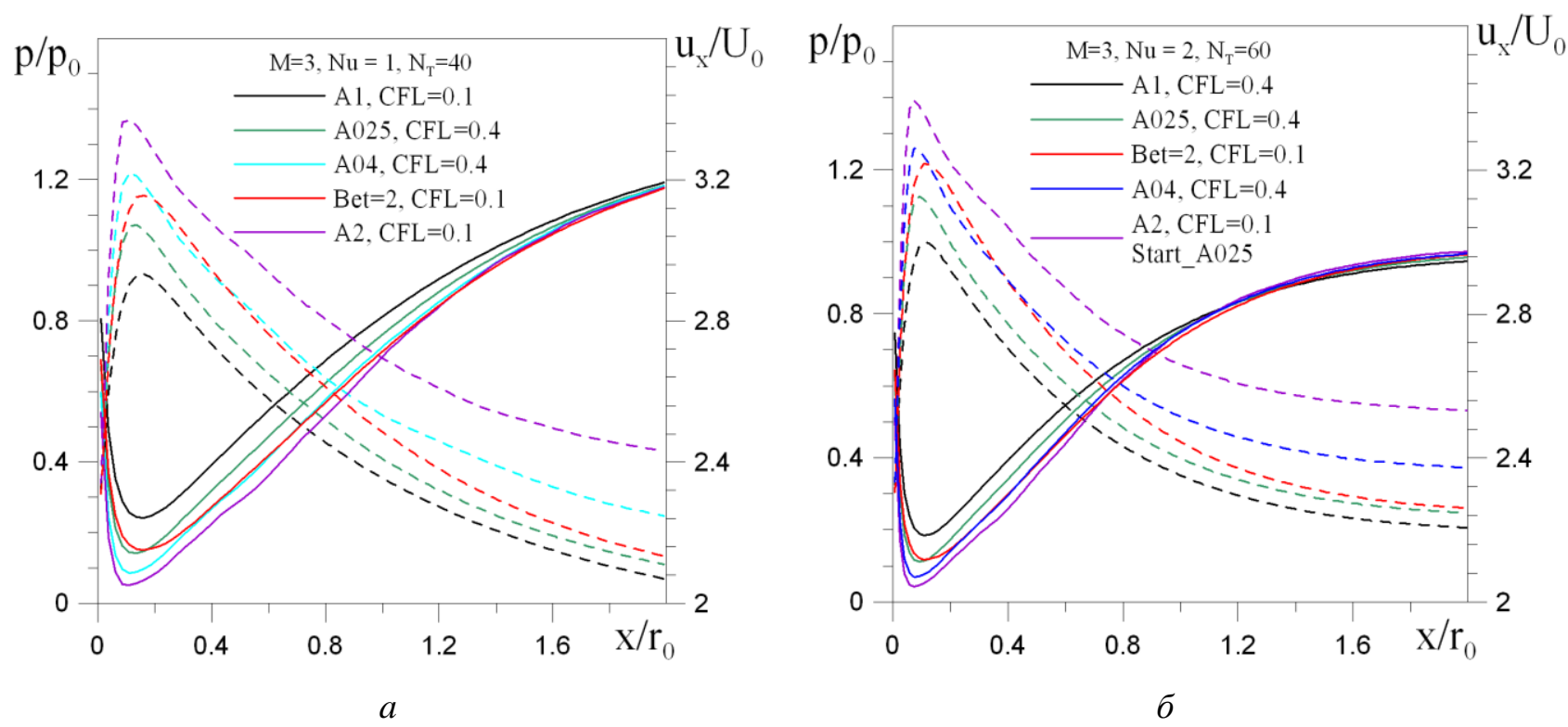

Рис. 8. Давление (сплошные) и продольная скорость (штриховые линии) на горизонтальной поверхности обтекаемых тел: $a$ - выступа при $N_{\mathrm{T}}=40 ; \sigma$ - кругового цилиндра $N_{\mathrm{T}}=60$

Априори можно полагать, что наиболее точными должны быть расчеты по схеме Колгана. На рис. 9 представлены значения погрешности по полной энтальпии

и функции энтропии

$$
\delta H=\frac{h+0.5\left(u_{x}^{2}+u_{y}^{2}\right)-H_{0}}{H_{0}}
$$

$$
\delta \Psi=\frac{p / p_{0}}{\left(\rho / \rho_{0}\right)^{\gamma}}-1
$$

в расчетах, соответствующих рис. 8 . В идеале $\delta H=0$, поскольку полная энтальпия в условиях стационарного течения должна иметь постоянное значение. Энтропия претерпевает неоднородные изменения при переходе через головную ударную волну, поэтому погрешность расчетов по энтропии следует оценивать по изменению $\delta \Psi$ на поверхности обтекаемого тела. 

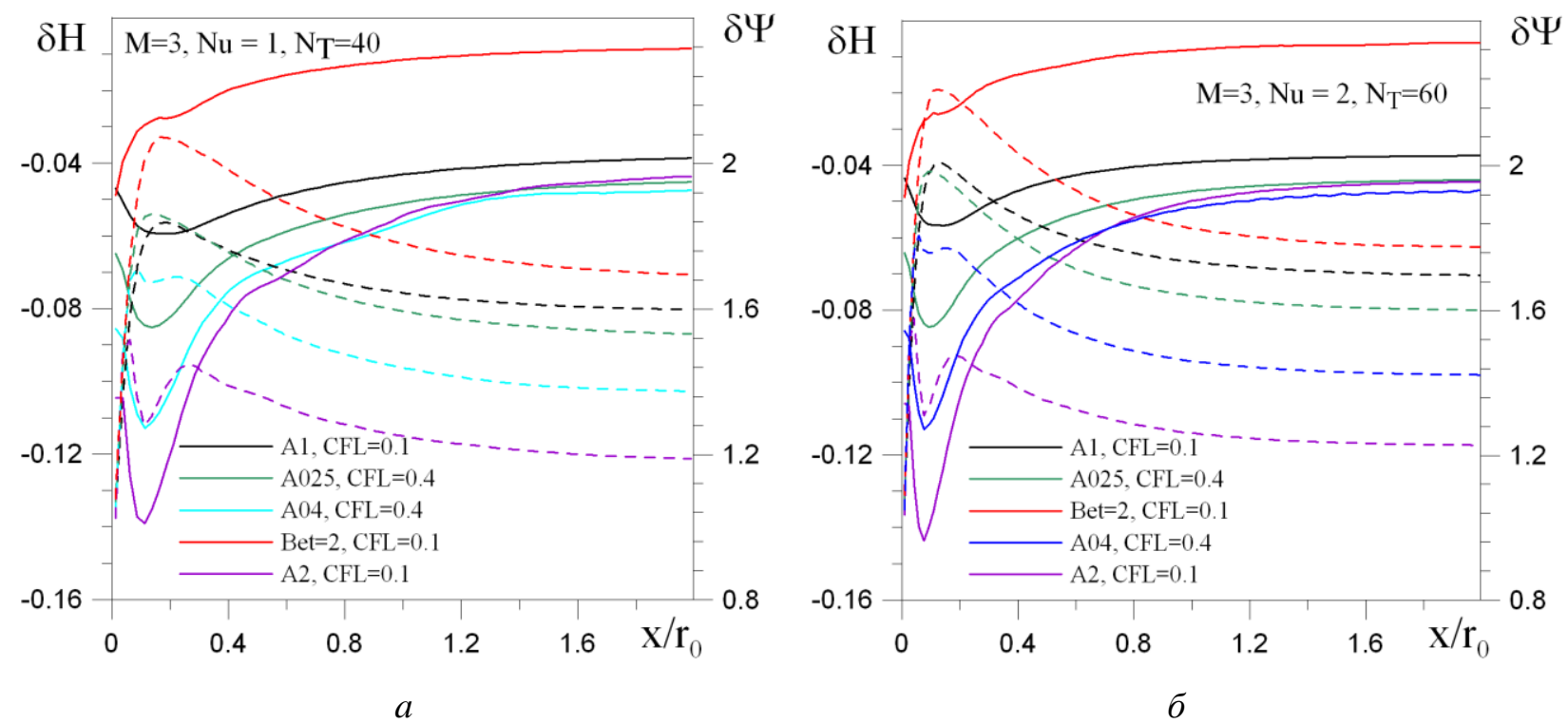

Рис. 9. Погрешность расчетов по полной энтальпии (сплошные) и энтропии (штриховые линии) на горизонтальной поверхности обтекаемых тел: $a$ - для выступа при $N_{\mathrm{T}}=40 ; \sigma$ - для кругового цилиндра при $N_{\mathrm{T}}=60$

По энтальпии наиболее точной оказывается схема Годунова-Колгана с переменной промежуточной точкой (рис. 9, кривые Bet $=2)$. Максимальная ошибка $(\sim 14 \%)$ получена в расчете по схеме Колгана. Пик $\delta \Psi$ в окрестности угловой точки $(x=0)$ связан с формированием упомянутой выше внутренней ударной волны SF над телом вслед за областью разрежения. Небольшая разница значений $\delta \Psi$ в точках $x=0$ и $x=2$ обусловлена невысокой интенсивностью этой ударной волны.

\section{3. Заключение}

1. Численно показано, что схема Колгана не гарантирует однозначного ответа на вопрос о режиме обтекания рассматриваемых тел сверхзвуковым потоком. Модификации схемы Годунова с промежуточной точкой способны моделировать установившееся обтекание плоского выступа и кругового цилиндра сверхзвуковым коаксиальным потоком.

2. Стационарные решения соответствуют результатам экспериментов, поэтому могут считаться физически обоснованными. Следует, однако, отметить, что такие режимы обтекания обеспечиваются схемной вязкостью, которая заменяет вязкость реального газа. Решения с наклонными ударными волнами, пульсирующими перед торцевой стенкой, не соответствуют экспериментальным результатам, но они не противоречат уравнениям Эйлера, поскольку обусловлены низкой схемной вязкостью.

\section{Благодарности и ссылки на гранты}

Работа выполнена с использованием суперкомпьютера МГУ «Ломоносов».

\section{Литература}

1. Годунов С.К. Разностный метод численного расчета разрывных решений уравнений гидродинамики// Математический сборник. 1959. Т. 47(89). № 3. С. 271-306.

2. Годунов С.К., Забродин А.В., Иванов М.Я., Крайко А.Н., Прокопов Г.П. Численное решение многомерных задач газовой динамики// М.: Наука, 1976. 400 с.

3. Колган В.П. Применение принципа минимальных значений производной к построению конечно-разностных схем для расчета разрывных решений газовой динамики// Ученые записки ЦАГИ. 1972. Т.III. № 6. С. 68-77. 
4. Колган В.П. Конечно-разностная схема для расчета двумерных разрывных решений нестационарной газовой динамики// Ученые записки ЦАГИ. 1975. Т.VI. № 1. С. 9-14.

5. Тилляева Н.И. Обобщение модифицированной схемы С.К. Годунова на произвольные нерегулярные сетки// Ученые записки ЦАГИ. 1986. Т.XVII. № 2. С. 18-26.

6. Harten A., Lax P. D., B. van Leer, On upstreaming differencing and Godunov-type schemes for hyperbolic conservation laws// SIAM review. Vol. 25, 1983. Pp.35-61.

7. Roe P.L. Approximate Riemann solvers, Parameter Vectors, and Difference Schemes // J. Comput. Phys. Vol. 43. 1981. Pp. 357-372.

8. Osher S., Chakravarthy S.R. Upwind schemes and boundary conditions with applications to Euler equations in general geometries // J. Comput. Phys. Vol. 50, 1983. Pp. 447-481.

9. Schulz-Rinne C. W., Collins J. P., Glaz H. M. Numerical solution of the Riemann problem for twodimensional gas dynamics// SIAM J. Sci. Comput., 14 (1993), pp. 1394-1414.

10. Toro B.F., Chakraborty A. Development of an approximate Riemann solver for the steady supersonic Euler equations // The Aeronautical Journal. Vol. 98, 1994. Pp. 325-339.

11. Васильев Е.И. W-модификация метода С.К. Годунова и ее применение для двумерных нестационарных течений запыленного газа// ЖВМиМФ. 1996. Т. 36. № 1. С. 122-135.

12. Tunik Yu. V. Instability of Contact Surface in Cylindrical Explosive Waves// Fluid Mech Open Acc. 2017. V. 4. No 4. DOI: 10.4172/2476-2296.1000168

13. Белоцерковский О.М., Давыдов Ю.М. Метод крупных частиц в газовой динамике//М.: Наука. Главная редакция физико-математической литературы. 1982. 392с.

14. Альбом течений жидкости и газа: А56/ Составитель М. Ван-Дайк. Москва: «Мир». 1986, - 184 с. (Перевод с английского). 